\title{
HSE hybrid functional within the FLAPW method and its application to GdN
}

\author{
Martin Schlipf, Markus Betzinger, Christoph Friedrich, Marjana Ležaić, and Stefan Blügel \\ Peter Grünberg Institut and Institute for Advanced Simulation, Forschungszentrum Jülich and JARA, D-52425 Jülich, Germany
}

(Received 4 July 2011; published 30 September 2011)

\begin{abstract}
We present an implementation of the Heyd-Scuseria-Ernzerhof (HSE) hybrid functional within the fullpotential linearized augmented-plane-wave (FLAPW) method. Pivotal to the HSE functional is the screened electron-electron interaction, which we separate into the bare Coulomb interaction and the remainder. Both terms give rise to exchange potentials, which sum up to the screened nonlocal exchange potential of HSE. We evaluate the former with the help of an auxiliary basis, defined in such a way that the bare Coulomb matrix becomes sparse. The latter, which is a slowly varying function in real space, is computed efficiently in reciprocal space. This approach is general and can be applied to a whole class of screened hybrid functionals. We obtain excellent agreement of band gaps and lattice constants for prototypical semiconductors and insulators with electronic-structure calculations using plane-wave or Gaussian basis sets. We apply the HSE hybrid functional to examine the ground-state properties of rocksalt $\mathrm{GdN}$, which have been controversially discussed in literature. Our results indicate that there is a half-metal to insulator transition occurring between the theoretically optimized lattice constant at $0 \mathrm{~K}$ and the experimental lattice constant at room temperature. Overall, we attain good agreement with experimental data for band transitions, magnetic moments, and the Curie temperature.
\end{abstract}

DOI: 10.1103/PhysRevB.84.125142

PACS number(s): 71.15.Ap, 71.15.Mb, 31.15.E-

\section{INTRODUCTION}

Density functional theory (DFT) $)^{1,2}$ is a powerful tool for calculating the electronic ground-state properties of molecules and solids. The predictive power of numerical DFT calculations relies on the availability of accurate approximations for the exchange-correlation (xc) energy $E_{\mathrm{xc}}$, which incorporates all complicated many-body effects. In many systems, this quantity is described adequately by the local-density approximation (LDA). ${ }^{3-5}$ However, in more complex systems, physical properties such as the geometric structure, magnetic properties, and the band gap are not well reproduced. One can go beyond the LDA by taking into account the local density gradient, which yields the generalized gradient approximation (GGA), ${ }^{6,7}$ upon which many functionals are based. However, despite their success, the GGA functionals still often fail in describing systems with localized states, which is attributed to an incomplete cancellation of the self-interaction error in these semilocal functionals..$^{8,9}$

This deficiency is particularly critical in systems whose electronic properties are largely governed by the correlated motion of electrons in localized states. The rare-earth chalcogenides are among this class of materials, having incompletely filled $f$-electron shells. They are insulating, semiconducting, or metallic depending on details of the valency of the rare-earth element. Gadolinium nitride ( $\mathrm{GdN}$ ) is widely studied owing to the ferromagnetic order, the large magnetic moment of $6.88 \mu_{\mathrm{B}}$ per Gd atom, ${ }^{10}$ and its large magnetoresistive effect, ${ }^{11}$ which makes the material interesting for technological applications. The mechanism of the ferromagnetic order is still under debate. Various types are being discussed, such as carrier mediated ${ }^{12,13}$ and superexchange mechanisms. ${ }^{14}$ Another point of debate is the electronic properties. It was experimentally demonstrated to be a low carrier semimetal in single crystals ${ }^{15}$ and insulating in thin films. ${ }^{16}$ There are also several recent reports of thin films of GdN having a degenerately doped semiconducting ${ }^{17-19}$ or a metallic ground state ${ }^{19}$ based on the resistivity data measured at low temperatures. Theoretically it is predicted to have a semiconducting ${ }^{20,21}$ or a half-metallic character ${ }^{14,22,23}$ based on $a b$ initio calculations.

Materials with strongly localized states, such as the $f$ states in $\mathrm{GdN}$, are often treated within the LSDA $+U$ method, ${ }^{24}$ where the electron correlation in these states is described with an additional on-site term, that involves the Hubbard parameter $U$. The disadvantage of this method is that the value of $U$ is not known a priori. Although methods to estimate the $U$ value from first-principles calculations have been developed, ${ }^{25-27}$ it is usually chosen to reproduce experimental observations. However, a specific $U$ value that provides a good description of one quantity is often not suitable to describe another quantity. ${ }^{28}$

During the last decade, hybrid functionals that combine a fraction of nonlocal Hartree-Fock (HF) exchange with local $\mathrm{xc}$ functionals have been shown to be a viable improvement over LDA and GGA offering a parameter-free description specifically suited for band-gap materials. ${ }^{29,30}$ The explicit consideration of nonlocal HF exchange leads to a partial cancellation of the self-interaction error, but also makes numerical calculations considerably more demanding than conventional LDA and GGA calculations. Various hybrid functionals have been developed. In empirical hybrid functionals, such as the B3LYP functional, ${ }^{31}$ the fraction of HF exchange is determined by fitting to an experimental data set. In the PBE0 functional ${ }^{32}$ the mixing parameter for the HF exchange is inferred from expanding the integrand of the adiabatic-connection formula of the exact xc functional.

In periodic systems, the Coulomb interaction between the electrons is effectively screened by polarization effects in the electron system. The effective interaction is particularly short-ranged in systems with small or vanishing band gaps. Therefore Heyd et al. ${ }^{33}$ introduced a range-separated hybrid functional, which has the added benefit to reduce the computational cost within a basis of localized Gaussian functions. Starting from the PBE0 functional, they partitioned the Fock exchange term into a short- and a long-range part, where the former is described by a correspondingly screened 
Fock term and the latter is treated by a local approximation, derived from the PBE functional. ${ }^{7}$ Heyd et al. showed that this hybrid functional leads to a reduced computational demand for localized basis sets compared with the PBE0 functional. Furthermore, it even yields results that are often in better agreement with experiment. ${ }^{30}$

The Heyd-Scuseria-Ernzerhof (HSE) hybrid functional has been implemented within Gaussian ${ }^{33}$ and plane-wave ${ }^{34,35}$ basis sets. In this work, we present an implementation within the full-potential linearized augmented-plane-wave (FLAPW) approach as implemented in the FLEUR code, ${ }^{36}$ which provides a highly accurate all-electron basis ${ }^{37-39}$ for a large variety of materials, including open systems with low symmetry, $d$ - and $f$-electron systems, as well as oxides and nitrides. An implementation of the PBE0 functional limited to certain localized states and on-site interactions was given by Tran et al. ${ }^{40}$ Betzinger et al. ${ }^{41}$ described an efficient way to calculate the full nonlocal exchange potential for the PBE0 functional without these restrictions. Very recently, Tran and Blaha $^{42}$ reported an implementation of hybrid functionals whose nonlocal exchange integrals are evaluated using the pseudocharge method of Weinert. ${ }^{43}$ However, this approach mathematically restricts the electron-electron interaction to potentials that are solutions of Laplace-type equations, whose radial solutions can be expressed as analytically or numerically known regular and irregular solutions and spherical harmonics, such as the bare Coulomb and the screened Yukawa potential. The error function used in the HSE functional does not have this property. In our implementation, there is no such restriction. Our approach is very general. In fact, any kind of interaction potential can be implemented for the nonlocal exchange potential by changing only a few lines of code. The only requirement is that it differs from the bare Coulomb potential by a function that possesses a fast Fourier expansion, a condition that is fulfilled by all physical screened potentials, including the error function used in the HSE functional.

Our numerical approach extends the implementation of Ref. 41, which is based on an auxiliary basis that is designed to represent products of wave functions. This so-called mixed product basis (MPB) is constructed directly from products of LAPW basis functions and retains the full accuracy of the all-electron description. Several techniques were introduced to accelerate the evaluation of the computationally expensive nonlocal exchange term. Spatial and time-reversal symmetries are exploited to restrict the Brillouin-zone (BZ) summations to irreducible sets of $\mathbf{k}$ points. The nonlocal potential is calculated in the basis of single-particle eigenstates, which allows to truncate the matrix at a certain number of bands. The divergence of the Coulomb potential in the BZ center is treated analytically instead of using dense $\mathbf{k}$-point sets around $\mathbf{k}=\mathbf{0}$. A nested density convergence scheme greatly reduces the number of iterations in the self-consistent field cycle. Finally, by a suitable transformation of the MPB the Coulomb matrix becomes sparse, which speeds up the matrix-vector operations considerably.

This transformation relies on the analytic properties of the bare Coulomb potential. Any other potential, in particular the screened Coulomb interaction, will not lead to a sparse matrix representation, though. Furthermore, in contrast to Gaussian or plane-wave basis sets, a direct evaluation of the screened Coulomb matrix, in the same way as for the bare Coulomb matrix, ${ }^{44}$ is cumbersome in the MPB. Therefore, we incorporate the screening, after calculating the bare nonlocal exchange potential, in a separate step, which produces hardly any overhead. In this way, the simple analytic properties of the bare Coulomb potential as well as the sparsity of the Coulomb matrix are retained and can be taken advantage of.

We validate our implementation by comparing results for prototypical semiconductors and insulators with results from the literature. Then, we calculate the ground-state properties and the band structure of GdN. The band gap of $\mathrm{GdN}$ is controversially discussed in literature. Results from LSDA $+U$ calculations are inconclusive. While the linearized muffin-tin orbital (LMTO) approach yields a narrow-gap semiconductor as ground state, ${ }^{12,20,45,46} \mathrm{GdN}$ exhibits a transition from a half-metallic to a semiconducting ground state under strain within the FLAPW method. ${ }^{14}$ Two different solutions close in energy were obtained in an investigation using the hybrid functional B3LYP ${ }^{23}$ Both solutions were half-metallic, one in the majority spin channel, the other one in the minority spin channel. Our results show a transition from a half-metallic ground state, which is similar to the energetically slightly less favorable solution of Ref. 23, to a semiconductor under strain as in the LSDA $+U$ calculation of Ref. 14. In their work, a large change of the lattice constant of more than $10 \%$ was necessary to observe this transition. However, our calculations indicate that already small volume changes $(\approx 0.5 \%)$ are sufficient to observe this transition.

The paper is organized as follows. In Sec. II, we give a brief introduction to the theory of hybrid functionals. In Sec. III, we introduce the FLAPW method and describe our implementation of the HSE functional. In Sec. IV, we first compare results for prototypical semiconductors and insulators with values from the literature. Then, we present our findings for GdN in Sec. V. In Sec. VI, we draw our conclusions.

\section{THEORY}

The construction of hybrid functionals as mixtures of local functionals with a nonlocal exchange term is motivated by the adiabatic-connection formula, $, 47,48$ in which the scaling of the electron-electron interaction establishes a connection between the noninteracting Kohn-Sham system with the fully interacting one. In the weakly interacting limit, the formula becomes identical to the HF exchange term, which prompted Becke $^{31,49}$ to introduce a certain fraction $a$ of HF exchange into the xc functional

$$
E_{\mathrm{xc}}^{\mathrm{HYB}}=E_{\mathrm{xc}}^{\mathrm{L}}+a\left(E_{\mathrm{x}}^{\mathrm{HF}}-E_{\mathrm{x}}^{\mathrm{L}}\right),
$$

where $E_{\mathrm{xc}}^{\mathrm{L}}$ denotes the local xc functional and $E_{\mathrm{x}}^{\mathrm{L}}$ its exchange part. $E_{\mathrm{x}}^{\mathrm{HF}}$ is the HF exchange energy

$$
\begin{aligned}
E_{\mathrm{x}}^{\mathrm{HF}}= & -\frac{1}{2} \sum_{\sigma} \sum_{\mathbf{k}, \mathbf{q}}^{\mathrm{BZ}} \sum_{n, n^{\prime}}^{\mathrm{occ}} \iint \mathrm{d}^{3} r \mathrm{~d}^{3} r^{\prime} \\
& \times \varphi_{n \mathbf{k}}^{\sigma *}(\mathbf{r}) \varphi_{n^{\prime} \mathbf{q}}^{\sigma}(\mathbf{r}) v\left(\left|\mathbf{r}-\mathbf{r}^{\prime}\right|\right) \varphi_{n^{\prime} \mathbf{q}}^{\sigma *}\left(\mathbf{r}^{\prime}\right) \varphi_{n \mathbf{k}}^{\sigma}\left(\mathbf{r}^{\prime}\right),
\end{aligned}
$$

evaluated with the Kohn-Sham wave functions $\varphi_{n \mathbf{k}}^{\sigma}(\mathbf{r})$ of spin $\sigma$, wave vector $\mathbf{k}$, and band index $n$. The sums over $\mathbf{k}$ and 
q run over the full Brillouin zone (BZ), $n$ and $n^{\prime}$ sum over all occupied states, and $v(r)=1 / r$ is the bare Coulomb potential. Here and in the following, atomic units are used unless stated otherwise. As the wave functions $\varphi_{n \mathbf{k}}^{\sigma}(\mathbf{r})$ are functionals of the effective potential, which in turn is a functional of the density, $E_{\mathrm{x}}^{\mathrm{HF}}$ is a true functional of the density, too.

Perdew et $a{ }^{32}$ deduced a mixing parameter $a=0.25$ by assuming a certain shape for the adiabatic-connection integrand. They proposed to use the Perdew-Burke-Ernzerhof (PBE) functional ${ }^{7}$ for the local part. The resulting functional

$$
E_{\mathrm{xc}}^{\mathrm{PBE} 0}=E_{\mathrm{xc}}^{\mathrm{PBE}}+a\left(E_{\mathrm{x}}^{\mathrm{HF}}-E_{\mathrm{x}}^{\mathrm{PBE}}\right)
$$

is nowadays referred to as PBE0.

As the long-range part of the nonlocal HF term is cumbersome to calculate, Heyd et al. ${ }^{33,50}$ suggested to replace it again by a simple local functional. Later, it was demonstrated ${ }^{30}$ that this leads to an improved description of the band gaps of semiconductors. Heyd et al. ${ }^{33}$ used the error function $\operatorname{erf}(x)$ and its complement $\operatorname{erfc}(x)=1-\operatorname{erf}(x)$ to decompose the Coulomb interaction into a long-range (LR) and a short-range (SR) part

$$
v(r)=\frac{\operatorname{erf}(\omega r)}{r}+\frac{\operatorname{erfc}(\omega r)}{r}=v^{\mathrm{LR}}(r)+v^{\mathrm{SR}}(r),
$$

where $\omega$ is an adjustable screening parameter. The HSE hybrid functional is thus given by

$$
E_{\mathrm{xc}}^{\mathrm{HSE}}(\omega)=E_{\mathrm{xc}}^{\mathrm{PBE}}+a\left[E_{\mathrm{x}}^{\mathrm{HF}, \mathrm{SR}}(\omega)-E_{\mathrm{x}}^{\mathrm{PBE}, \mathrm{SR}}(\omega)\right],
$$

where $E_{\mathrm{x}}^{\mathrm{HF}, \mathrm{SR}}(\omega)$ corresponds to Eq. (2) with the bare Coulomb potential $v(r)$ replaced by $v^{\mathrm{SR}}(r) . E_{\mathrm{x}}^{\mathrm{PBE}, \mathrm{SR}}(\omega)$ is the local functional for the SR exchange according to the decomposition given in Eq. (4). Its numerical treatment is discussed in Refs. 33 and 51. Based on numerical fits to benchmark data sets of molecules, Heyd et al. ${ }^{33}$ found an optimized value for the screening parameter $\omega=0.15$. In this work, we employ the value of $\omega=0.11$, which was optimized for solids. ${ }^{52}$

Hybrid functionals are usually applied within the generalized Kohn-Sham formalism, ${ }^{53}$ which is based on a fictitious system of noninteracting electrons. These particles move subject to a nonlocal potential that is defined in such a way that the electron density $n(\mathbf{r})$ equals that of the real system. The nonlocal potential contains a local part that consists of the external potential created by the nuclear charges, the Hartree potential, i.e., the electrostatic potential produced by the total electron charge density, and a local contribution that derives from functional differentiation of the local parts of Eqs. (3) and (5) for the PBE0 and HSE functionals, respectively. The implementation of this local part of the xc potential requires only minor modifications of the DFT code, and we will focus on the nonlocal part in the following, which derives from the nonlocal exchange energy functional $E_{\mathrm{x}}^{\mathrm{HF}}$. Leaving out the scaling factor $a$, its matrix representation in the basis of Kohn-Sham eigenstates is given by

$$
\begin{aligned}
V_{\mathrm{x}, n n^{\prime}}^{\sigma, \mathrm{NL}}(\mathbf{k})= & -\sum_{\mathbf{q}}^{\mathrm{BZ}} \sum_{m}^{\mathrm{occ}} \iint \mathrm{d}^{3} r \mathrm{~d}^{3} r^{\prime} \\
& \times \varphi_{n \mathbf{k}}^{\sigma *}(\mathbf{r}) \varphi_{m \mathbf{q}}^{\sigma}(\mathbf{r}) v\left(\left|\mathbf{r}-\mathbf{r}^{\prime}\right|\right) \varphi_{m \mathbf{q}}^{\sigma *}\left(\mathbf{r}^{\prime}\right) \varphi_{n^{\prime} \mathbf{k}}^{\sigma}\left(\mathbf{r}^{\prime}\right) .
\end{aligned}
$$

For the HSE functional, the bare interaction $v(r)$ would have to be replaced by the screened interaction $v^{\mathrm{SR}}(r)$. Yet, in practice, we first evaluate the nonlocal potential in the form of Eq. (6) and correct for the screening afterwards by subtracting $v^{\mathrm{LR}}(r)$, as will be explained in the next section.

\section{IMPLEMENTATION}

\section{A. Basis sets}

Our implementation of the HSE functional is based on the all-electron FLAPW method, ${ }^{37-39}$ in which space is partitioned into nonoverlapping atom-centered muffin-tin (MT) spheres and the remaining interstitial region (IR). The core states, which are confined to the spheres, are obtained from solving the fully relativistic Dirac equation. For the valence and conduction states we use a set of basis functions that are defined differently in the two regions of space: plane waves $\mathrm{e}^{\mathrm{i}(\mathbf{k}+\mathbf{G}) \cdot \mathbf{r}}$ with $|\mathbf{k}+\mathbf{G}| \leqslant G_{\max }$ in the IR and linear combinations of $u_{l p}^{a \sigma}(r) Y_{l m}(\widehat{\mathbf{r}})$ in the MT spheres, where $r$ is measured from the sphere center, $u_{l p}^{a \sigma}(r)$ are numerical functions defined on a radial grid, $Y_{l m}(\widehat{\mathbf{r}})$ are spherical harmonics with angularmomentum quantum numbers $0 \leqslant l \leqslant l_{\max }$ and $|m| \leqslant l$, $a$ labels the atom in the unit cell, and $p$ is an index for different radial functions. $G_{\max }$ and $l_{\max }$ are cutoff parameters. The linear combinations are such that the basis functions and their radial derivatives are continuous at the MT sphere boundaries.

As in Ref. 41, we evaluate the nonlocal potential, Eq. (6), with the help of an auxiliary basis $\left\{M_{\mathbf{q} J}(\mathbf{r})\right\}$ and its biorthogonal set $\left\{\tilde{M}_{\mathbf{q} J}(\mathbf{r})\right\}$, where $\mathbf{q}$ is a Bloch vector and $J$ is used to index these basis functions. By placing the completeness relation

$$
1=\sum_{\mathbf{q} J}\left|M_{\mathbf{q} J}\right\rangle\left\langle\tilde{M}_{\mathbf{q} J}\left|=\sum_{\mathbf{q} J}\right| \tilde{M}_{\mathbf{q} J}\right\rangle\left\langle M_{\mathbf{q} J}\right|
$$

at both sides of $v(r)$, the six-dimensional integral becomes a sum over vector-matrix-vector products in the space of the MPB

$$
\begin{aligned}
V_{\mathrm{x}, n n^{\prime}}^{\sigma, \mathrm{NL}}(\mathbf{k})= & -\sum_{m}^{\text {occ. }} \sum_{\mathbf{q}}^{\mathrm{BZ}} \sum_{I J}\left\langle\varphi_{n \mathbf{k}}^{\sigma} \mid \varphi_{m \mathbf{k}-\mathbf{q}}^{\sigma} M_{\mathbf{q}, I}\right\rangle \\
& \times v_{I J}(\mathbf{q})\left\langle M_{\mathbf{q}, J} \varphi_{m \mathbf{k}-\mathbf{q}}^{\sigma} \mid \varphi_{n^{\prime} \mathbf{k}}^{\sigma}\right\rangle,
\end{aligned}
$$

with the usual bra-ket notation $\langle f \mid g\rangle=\int \mathrm{d}^{3} r f^{*}(\mathbf{r}) g(\mathbf{r})$ and the Coulomb matrix ${ }^{44}$

$$
v_{I J}(\mathbf{q})=\iint \mathrm{d}^{3} r \mathrm{~d}^{3} r^{\prime} \tilde{M}_{\mathbf{q}, I}^{*}(\mathbf{r}) v\left(\mathbf{r}, \mathbf{r}^{\prime}\right) \tilde{M}_{\mathbf{q}, J}\left(\mathbf{r}^{\prime}\right) .
$$

We need to evaluate the latter only once at the beginning of the self-consistency cycle. We note here again that the screening is accounted for in a separate step [cf. Eq. (11)].

As Eq. (8) indicates, the auxiliary basis must be sufficiently complete in the space of wave-function products. We therefore construct this basis directly from products of LAPW basis functions. In the interstitial region, the auxiliary basis functions are plane waves with a new cutoff parameter $G_{\max }^{\prime}$. In the MT spheres, the basis consists of numerical functions of the form $M_{L P}^{a}(r) \mathrm{Y}_{L M}(\widehat{\mathbf{r}})$ with a cutoff $L_{\max }$. This so-called mixed product basis (MPB) can be systematically converged to represent the products of LAPW wave functions numerically exactly. In this way, the MPB is on the same level of accuracy 
as the all-electron LAPW basis for the wave functions. It was shown ${ }^{41,54}$ that $G_{\max }^{\prime}$ and $L_{\max }$ can be well below their mathematically determined exact limit, i.e., $2 G_{\max }$ and $2 l_{\max }$, and even below $G_{\max }$ and $l_{\max }$, which makes the MPB a very efficient basis. Further details about the MPB can be found elsewhere. $^{41,44,54}$

\section{B. Implementation of the HSE functional}

It seems that the implementation of the HSE functional is now straightforward: the bare Coulomb potential in Eq. (9) is replaced by the screened one and it is proceeded as in Ref. 41 for the case of the PBE0 functional. However, in this way we would loose a very favorable property of the bare Coulomb potential, namely, its multipole expansion, which makes it possible to render the Coulomb matrix sparse by a simple unitary transformation of the MPB. The sparsity of $v_{I J}(\mathbf{q})$ speeds up the matrix-vector multiplications in Eq. (8) considerably. The screened Coulomb potential does not have this simple property. Furthermore, the direct evaluation of $v_{I J}(\mathbf{q})$ following the techniques of Ref. 44 is not transferable to the screened interaction.

For these reasons, we evaluate Eq. (8) with the bare interaction as before and use a separate step to incorporate the screening. This procedure is motivated by Fig. 1, which shows the bare and screened Coulomb potentials, $v(r)$ and $v^{\mathrm{SR}}(r)$, as well as the difference, $v^{\mathrm{LR}}(r)$, as a function of the distance $r$, measured in units of Bohr radii $a_{0}$. While the two potentials diverge at $r=0$, their difference remains finite. It has a very smooth behavior for all distances and should thus be suitable to be described in reciprocal space. In fact, we find that only very few plane waves are needed to represent the difference accurately.

To make use of the quickly converging Fourier expansion of the long-range potential $v^{\mathrm{LR}}(r)$, we rewrite the total xc potential in the form

$$
V_{\mathrm{xc}}^{\mathrm{HSE}}=V_{\mathrm{xc}}^{\mathrm{PBE}}-a V_{\mathrm{x}}^{\mathrm{PBE}, \mathrm{SR}}+a\left(V_{\mathrm{x}}^{\mathrm{NL}}-V_{\mathrm{x}}^{\mathrm{NL}, \mathrm{LR}}\right)
$$

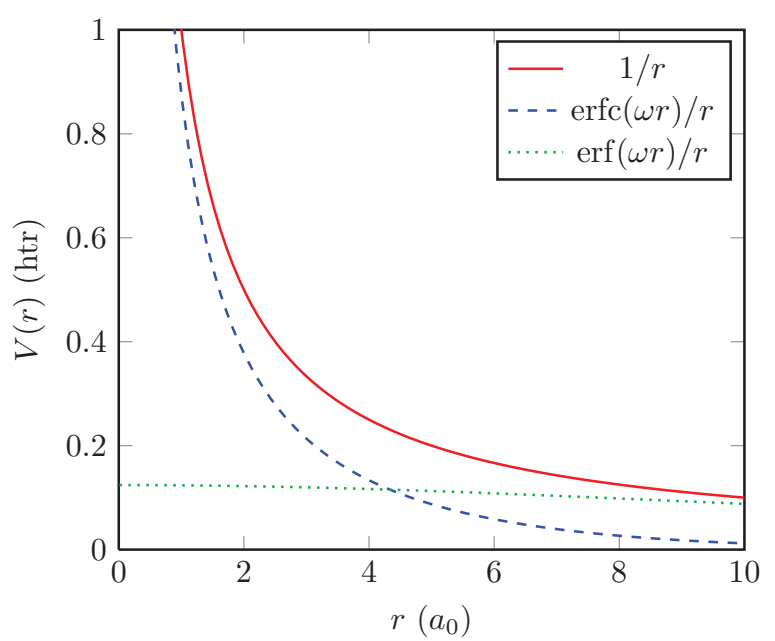

FIG. 1. (Color online) Comparison of the bare (red solid line) and the screened (blue dashed line) Coulomb potentials with the difference between both (green dotted line). The difference does not exhibit a divergence at $r=0$ and is a smooth function everywhere. Its Fourier transform converges very rapidly. with the local xc potentials $V_{\mathrm{xc}}^{\mathrm{PBE}}$ and $V_{\mathrm{x}}^{\mathrm{PBE}, \mathrm{SR}}$ that derive from $E_{\mathrm{xc}}^{\mathrm{PBE}}$ and $E_{\mathrm{x}}^{\mathrm{PBE}, \mathrm{SR}}$, respectively, and the potential terms in the parenthesis sum up to the SR nonlocal potential for HSE as

$$
\begin{aligned}
V_{\mathrm{x}, n n^{\prime}}^{\sigma, \mathrm{NL}, \mathrm{SR}}(\mathbf{k})= & V_{\mathrm{x}, n n^{\prime}}^{\sigma, \mathrm{NL}}(\mathbf{k})+\sum_{m}^{\mathrm{occ}} \sum_{\mathbf{q}}^{\mathrm{BZ}} \sum_{\mathbf{G}}\left\langle\varphi_{n \mathbf{k}}^{\sigma} \mid \varphi_{m \mathbf{k}-\mathbf{q}}^{\sigma} \chi_{\mathbf{q}+\mathbf{G}}\right\rangle \\
& \times\left\langle\chi_{\mathbf{q}+\mathbf{G}}\left|v^{\mathrm{LR}}\right| \chi_{\mathbf{q}+\mathbf{G}}\right\rangle\left\langle\chi_{\mathbf{q}+\mathbf{G}} \varphi_{m \mathbf{k}-\mathbf{q}}^{\sigma} \mid \varphi_{n^{\prime} \mathbf{k}}^{\sigma}\right\rangle,
\end{aligned}
$$

where $\chi_{\mathbf{q}+\mathbf{G}}(\mathbf{r})=\mathrm{e}^{\mathrm{i}(\mathbf{q}+\mathbf{G}) \cdot \mathbf{r}} / \sqrt{V}$ is a plane wave normalized by the crystal volume $V$. We evaluate the Fourier transform of the wave-function products with the help of the MPB

$$
\left\langle\varphi_{n \mathbf{k}}^{\sigma} \mid \varphi_{m \mathbf{k}-\mathbf{q}}^{\sigma} \chi_{\mathbf{q}+\mathbf{G}}\right\rangle=\sum_{I}\left\langle\varphi_{n \mathbf{k}}^{\sigma} \mid \varphi_{m \mathbf{k}-\mathbf{q}}^{\sigma} M_{\mathbf{q} I}\right\rangle\left\langle\tilde{M}_{\mathbf{q} I} \mid \chi_{\mathbf{q}+\mathbf{G}}\right\rangle
$$

where the first integrals on the right-hand side are calculated routinely already for $V_{\mathrm{x}, n n^{\prime}}^{\sigma, \mathrm{NL}}$. The Fourier transform of $v^{\mathrm{LR}}$ is known analytically

$$
\left\langle\chi_{\mathbf{q}+\mathbf{G}}\left|v^{\mathrm{LR}}\right| \chi_{\mathbf{q}+\mathbf{G}^{\prime}}\right\rangle=\frac{4 \pi}{|\mathbf{q}+\mathbf{G}|^{2}} \mathrm{e}^{-|\mathbf{q}+\mathbf{G}|^{2} / 4 \omega^{2}} \delta_{\mathbf{G G}^{\prime}} .
$$

We note that any other form of the screened Coulomb interaction could easily be implemented at this stage. Since the matrix elements are diagonal in reciprocal space, the second term of Eq. (11) takes in practical terms negligible time to compute. From the fact that this function approaches zero very quickly with $|\mathbf{q}+\mathbf{G}|$, it is clear that the results are easily converged up to machine precision, even if the Fourier coefficient in Eq. (12) falls off slowly with respect to $|\mathbf{q}+\mathbf{G}|$ because of the rapidly varying all-electron wave functions. Figure 2 shows the root-mean-square deviation of the eigenvalues of the matrix $V_{\mathrm{x}, n n^{\prime}}^{\sigma, \mathrm{NL}, \mathrm{SR}}(\mathbf{k})-V_{\mathrm{x}, n n^{\prime}}^{\sigma, \mathrm{NL}}(\mathbf{k})$, as a function of the number of $\mathbf{G}$ vectors used for its construction. The convergence was studied for the case of bulk silicon using a supercell with eight atoms. Machine precision is achieved with as few as 40 plane waves which would translate to ten

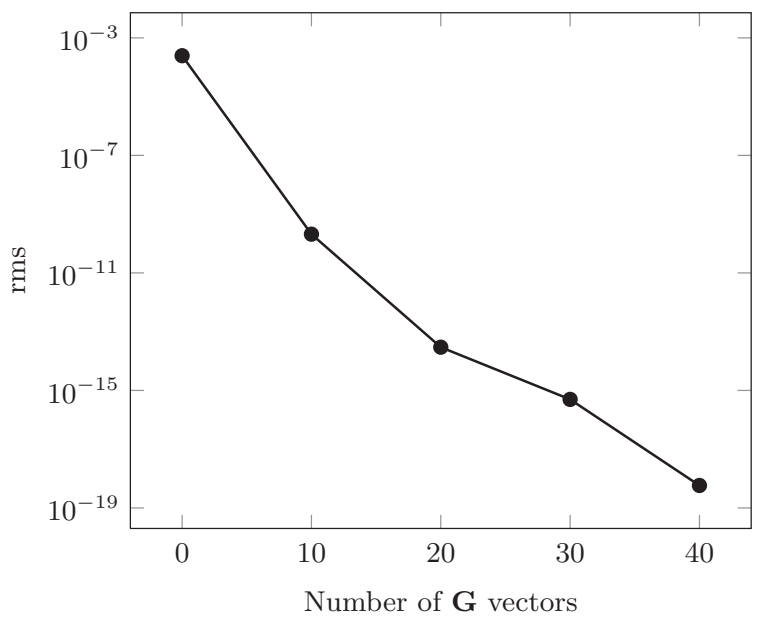

FIG. 2. Root-mean-square (rms) deviation of the eigenvalues of the second term in Eq. (11) from the fully converged result as a function of the number of plane waves in the Fourier transformation for the $\Gamma$ point of silicon. We have used a supercell containing eight atoms. The 40 Fourier components would translate to ten in a calculation of the primitive unit cell with two atoms. 
for a primitive unit cell containing two atoms. This behavior is independent of the $\mathbf{q}$ point.

We note that the Fourier transform in Eq. (13) diverges as $1 /|\mathbf{q}+\mathbf{G}|^{2}$ in the limit $\mathbf{q}+\mathbf{G} \rightarrow \mathbf{0}$. The same divergence is found for the bare Coulomb potential, ${ }^{41,44}$ such that the $1 /|\mathbf{q}+\mathbf{G}|^{2}$ terms cancel in the difference. The remainder is finite and is given by

$$
\lim _{\mathbf{q}+\mathbf{G} \rightarrow \mathbf{0}} \frac{4 \pi}{|\mathbf{q}+\mathbf{G}|^{2}}\left(1-\mathrm{e}^{-|\mathbf{q}+\mathbf{G}|^{2} / 4 \omega^{2}}\right)=\frac{\pi}{\omega^{2}} .
$$

We will later see that this nondivergent behavior of the screened interaction gives rise to a favorable k-point convergence.

\section{VERIFICATION}

First, we present calculations for a prototypical set of semiconductors (C, $\mathrm{Si}$, and $\mathrm{GaAs})$ and insulators ( $\mathrm{MgO}, \mathrm{NaCl}$, and $\mathrm{Ar}$ ) and compare the results with previous works from the literature. ${ }^{35,52}$ We focus in particular on direct and indirect band transitions. These are calculated as the energy differences of the highest occupied and the lowest unoccupied eigenstates at the corresponding points in the BZ. We have taken the experimental lattice constants from Ref. 55. In Fig. 3, we show the convergence of the band gap for silicon with the size of the k-point mesh. Within HSE this convergence is almost as fast as in PBE, whereas PBE0 requires larger $\mathbf{k}$-point meshes. This can be attributed to the nondivergent behavior of the screened interaction at $\mathbf{k}=\mathbf{0}$ (see Sec. III) and was already observed in Ref. 35 . We find that an $8 \times 8 \times 8 \mathbf{k}$-point mesh gives reliable HSE results for the band gap as well as for the ground-state properties.

In Table I, we compare our results for the $\Gamma \rightarrow \Gamma, \Gamma \rightarrow \mathrm{X}$, and $\Gamma \rightarrow \mathrm{L}$ transition energies with those obtained by the projector-augmented-wave (PAW) method ${ }^{35}$ and experimental data. The band transitions are calculated for a set of materials at their experimental lattice constants with the functionals PBE and HSE. Overall, we observe excellent agreement between the calculated values. In comparison to the experimental results, the HSE functional considerably improves on the PBE values.

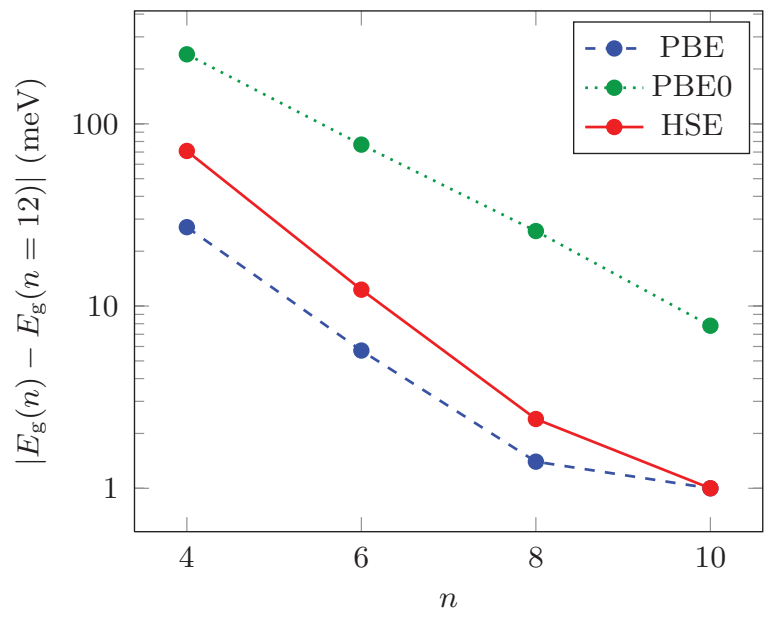

FIG. 3. (Color online) Convergence of the silicon band gap $E_{\mathrm{g}}$ for the functionals PBE (blue, dashed), PBE0 (green, dotted), and HSE (red, solid) with respect to the k-point mesh $(n \times n \times n)$.
TABLE I. Kohn-Sham transition energies in eV obtained with the functionals PBE and HSE at experimental lattice constants compared with values from PAW calculations and experiment. An $8 \times 8 \times$ 8 k-point mesh was employed.

\begin{tabular}{|c|c|c|c|c|c|c|}
\hline & & \multicolumn{2}{|c|}{ This work } & \multicolumn{2}{|c|}{ PAW $^{\mathrm{a}}$} & \multirow[t]{2}{*}{ Expt. } \\
\hline & & PBE & HSE & PBE & HSE & \\
\hline \multirow[t]{3}{*}{ GaAs } & $\Gamma \rightarrow \Gamma$ & 0.54 & 1.43 & 0.56 & 1.45 & $1.52,^{\mathrm{b}} 1.63^{\mathrm{c}}$ \\
\hline & $\Gamma \rightarrow \mathrm{X}$ & 1.47 & 2.06 & 1.46 & 2.02 & $1.90,{ }^{\mathrm{b}} 2.01,{ }^{\mathrm{c}} 2.18^{\mathrm{c}}$ \\
\hline & $\Gamma \rightarrow \mathrm{L}$ & 1.01 & 1.78 & 1.02 & 1.76 & $1.74,^{\mathrm{b}} 1.84,^{\mathrm{c}} 1.85^{\mathrm{c}}$ \\
\hline \multirow[t]{3}{*}{$\mathrm{Si}$} & $\Gamma \rightarrow \Gamma$ & 2.56 & 3.32 & 2.57 & 3.32 & $3.05,{ }^{\mathrm{d}} 3.34-3.36,{ }^{\mathrm{e}} 3.4^{\mathrm{c}}$ \\
\hline & $\Gamma \rightarrow \mathrm{X}$ & 0.71 & 1.29 & 0.71 & 1.29 & $1.13,{ }^{\mathrm{e}} 1.25^{\mathrm{d}}$ \\
\hline & $\Gamma \rightarrow \mathrm{L}$ & 1.54 & 2.24 & 1.54 & 2.24 & $2.06,{ }^{\mathrm{f}} 2.40^{\mathrm{c}}$ \\
\hline \multirow[t]{3}{*}{$\mathrm{C}$} & $\Gamma \rightarrow \Gamma$ & 5.60 & 6.98 & 5.59 & 6.97 & $7.3^{\mathrm{b}}$ \\
\hline & $\Gamma \rightarrow \mathrm{X}$ & 4.75 & 5.90 & 4.76 & 5.91 & \\
\hline & $\Gamma \rightarrow \mathrm{L}$ & 8.46 & 10.02 & 8.46 & 10.02 & \\
\hline \multirow[t]{3}{*}{$\mathrm{MgO}$} & $\Gamma \rightarrow \Gamma$ & 4.77 & 6.49 & 4.75 & 6.50 & $7.7^{\mathrm{g}}$ \\
\hline & $\Gamma \rightarrow \mathrm{X}$ & 9.14 & 10.86 & 9.15 & 10.92 & \\
\hline & $\Gamma \rightarrow \mathrm{L}$ & 7.93 & 9.69 & 7.91 & 9.64 & \\
\hline \multirow[t]{3}{*}{$\mathrm{NaCl}$} & $\Gamma \rightarrow \Gamma$ & 5.20 & 6.57 & 5.20 & 6.55 & $8.5^{\mathrm{h}}$ \\
\hline & $\Gamma \rightarrow X$ & 7.58 & 9.05 & 7.60 & 8.95 & \\
\hline & $\Gamma \rightarrow \mathrm{L}$ & 7.30 & 8.66 & 7.32 & 8.67 & \\
\hline $\mathrm{Ar}$ & $\Gamma \rightarrow \Gamma$ & 8.70 & 10.36 & 8.68 & 10.34 & $14.2^{\mathrm{i}}$ \\
\hline
\end{tabular}

${ }^{\mathrm{a}}$ Reference 35 .

${ }^{\mathrm{b}}$ Reference 56.

${ }^{\mathrm{c}}$ Reference 57.

${ }^{\mathrm{d}}$ Reference 58.

${ }^{\text {e}}$ Reference 59.

${ }^{\mathrm{f}}$ Reference 60.

${ }^{g}$ Reference 61.

${ }^{\mathrm{h}}$ Reference 62.

${ }^{\mathrm{i}}$ Reference 63.

For semiconductors, the HSE transition energies are in very good agreement with experiment, while the larger gaps of insulators are still underestimated.

We compute the equilibrium lattice constants and bulk moduli by calculating total energies for different lattice constants and fitting the results to the Murnaghan equation of state. ${ }^{64}$ In Tables II and III, we compare the lattice constants and bulk moduli obtained with our implementation of the HSE functional with results from implementations based on

TABLE II. Optimized lattice constants in $\AA$ obtained with the PBE and the HSE functional. An $8 \times 8 \times 8 \mathbf{k}$-point mesh was employed. Results are compared to experimental results and calculations using the HSE functional within a PAW ${ }^{35}$ and a Gaussian ${ }^{52}$ method.

\begin{tabular}{lccccc}
\hline \hline \multirow{2}{*}{ Functional } & \multicolumn{2}{c}{ This work } & & PAW & \\
\cline { 2 - 3 } & PBE & HSE & HSE & HSE & \\
\hline GaAs & 5.743 & 5.660 & 5.687 & 5.710 & 5.648 \\
$\mathrm{Si}$ & 5.472 & 5.441 & 5.435 & 5.451 & 5.430 \\
$\mathrm{C}$ & 3.571 & 3.549 & 3.549 & 3.557 & 3.567 \\
$\mathrm{MgO}$ & 4.265 & 4.217 & 4.210 & 4.222 & 4.207 \\
$\mathrm{NaCl}$ & 5.703 & 5.627 & 5.659 & 5.645 & 5.595 \\
\hline \hline
\end{tabular}

${ }^{\mathrm{a}}$ Reference 35 .

${ }^{\mathrm{b}}$ Reference 52 .

${ }^{\mathrm{c}}$ Experimental data taken from Ref. 55. 
TABLE III. Bulk moduli in GPa obtained with the PBE and the HSE functional. An $8 \times 8 \times 8$ k-point mesh was employed. Results are compared to experimental results and calculations using the HSE functional within a PAW method. ${ }^{35}$

\begin{tabular}{|c|c|c|c|c|}
\hline \multirow[b]{2}{*}{ Functional } & \multicolumn{2}{|c|}{ This work } & \multirow{2}{*}{$\begin{array}{c}\text { PAW }^{\mathrm{a}} \\
\text { HSE }\end{array}$} & \multirow[t]{2}{*}{ Expt. ${ }^{b}$} \\
\hline & PBE & HSE & & \\
\hline GaAs & 64.5 & 79.2 & 70.9 & 75.6 \\
\hline $\mathrm{Si}$ & 88.9 & 98.0 & 97.7 & 99.2 \\
\hline $\mathrm{C}$ & 433 & 467 & 467 & 443 \\
\hline $\mathrm{MgO}$ & 153 & 177 & 169 & 165 \\
\hline $\mathrm{NaCl}$ & 21.3 & 28.8 & 24.5 & 26.6 \\
\hline
\end{tabular}

a Reference 35 .

${ }^{\mathrm{b}}$ Experimental data taken from Ref. 55.

plane-wave $(\mathrm{PAW})^{35}$ and Gaussian basis sets. ${ }^{52}$ The results of all three methods agree very well. For example the lattice constants calculated in the FLAPW and PAW methods differ by less than 3 pm. Except for diamond, the HSE functional yields lattice constants and bulk moduli in much better agreement with experiment than the semilocal PBE functional, which tends to overestimate the former and underestimate the latter.

\section{GADOLINIUM NITRIDE}

\section{A. Computational setup}

GdN crystallizes in the rocksalt structure, with a roomtemperature lattice constant of $a_{\mathrm{GdN}}=4.988 \AA{ }^{70}$ The valence band of this material consists of the $\mathrm{N} 2 s, 2 p$, and the $\mathrm{Gd} 4 f$ states. The $4 f$ states are only half-occupied. The conduction band is formed by the Gd $5 d$ and $6 s$ states as well as the $4 f$ states in the minority channel.

We determine the numerical cutoff parameters for the calculations in such a way that the difference between the total energies calculated at the experimental lattice constant $a_{\mathrm{GdN}}$ and at $1.01 a_{\mathrm{GdN}}$ changes by less than $1 \mathrm{meV}$ upon increasing the parameters. In Table IV, we list the parameters used for the GdN unit cell (consisting of two atoms). In particular, we converged the k-point sampling, the size of the FLAPW basis, the size of the MPB, and the number of local orbitals. The latter are additional basis functions that are used to describe semicore states ${ }^{72}$ or to eliminate the linearization error. ${ }^{73,74}$

In the following, we compare our theoretical HSE results for the lattice constant, bulk modulus, band gaps, and magnetic moment with previous calculations and experiment. In order

TABLE IV. Numerical parameters used for the calculation of GdN.

\begin{tabular}{lcc}
\hline \hline \multicolumn{1}{c}{ Parameter } & \multicolumn{2}{c}{ Value } \\
\hline k-point mesh & $8 \times 8 \times 8$ & \\
muffin-tin radii & $R_{\mathrm{MT}}(\mathrm{Gd})=2.33 a_{0}$ & $R_{\mathrm{MT}}(\mathrm{N})=1.95 a_{0}$ \\
plane-wave cutoffs & $G_{\max }=4.9 a_{0}^{-1}$ & $G_{\max }^{\prime}=3.6 a_{0}^{-1}$ \\
angular-momentum & $l_{\max }(\mathrm{Gd})=12$ & $l_{\max }(\mathrm{N})=10$ \\
cutoffs & $L_{\max }(\mathrm{Gd})=6$ & $L_{\max }(\mathrm{N})=4$ \\
local orbitals ${ }^{\mathrm{a}}$ & $\mathrm{Gd}: 5 s, 5 p ; 7 s, 7 p, 6 d, 5 f$ & \\
& $\mathrm{~N}: 3 s, 3 p, 4 d, 5 f$ & \\
number of bands & 200 \\
\hline \hline
\end{tabular}

${ }^{\mathrm{a}}$ Reference 65 . to compare our band structure results obtained at $0 \mathrm{~K}$ with the experimental results obtained at room-temperature, where $\mathrm{GdN}$ is in the paramagnetic state, we follow the idea that there is no difference between the ferromagnetic and paramagnetic state for the exchange splitting and the large magnetic moments of the $4 f$ electrons. In the paramagnetic state we rather assume local magnetic $f$ moments that fluctuate in direction with an overall zero magnetization. Thus the magnetic polarization of the $\mathrm{N}$ states disappears. Dispersive valence and conduction electrons that exhibit a large group velocity feel at any moment in time a small random potential landscape due to the exchange potentials of $\mathrm{Gd} f$ moments pointing in random directions. Following Ref. 46 this can be approximated assuming that in the paramagnetic phase each of these $s, p$, and $d$ valence and conduction states characterized by a k-point band index are obtained by the averages of the corresponding spin-up and spin-down energies in the ferromagnetic phase.

Furthermore, from the total-energy differences we derive the exchange coupling constants for a Heisenberg spin Hamiltonian and determine the Curie temperature. This gives us a measure for the quality of energy differences that can be expected from the HSE functional between different magnetic states.

\section{B. Structural and electronic properties}

We start our investigation of $\mathrm{GdN}$ by evaluating its structural and electronic properties and comparing them to some of the available experimental ${ }^{10,68-70}$ and theoretical data, obtained with the hybrid B3LYP functional ${ }^{23}$ and within the LSDA $+U$ approach. ${ }^{14,46,68}$ The comparison is shown in Table V. We note that our parameter-free HSE calculations yield a lattice parameter of $4.967 \AA$ in very close agreement to the experiment, while B3LYP overestimates the value by $\sim 2 \%$ and the lattice constant in the LSDA $+U$ method depends on the choice of the parameter $U$. Thermal expansion could account for the remaining difference to the experimental lattice parameter that was determined at the room temperature (whereas the theoretical result corresponds to $0 \mathrm{~K}$ ). The thermal expansion coefficient of $\mathrm{GdN}$ is unknown so far. Assuming linear expansion between $0 \mathrm{~K}$ and room temperature $(293 \mathrm{~K})$ with the coefficient of isostructural and isovalent $\mathrm{EuO}\left(\approx 13 \times 10^{-6} \mathrm{~K}^{-1}\right),{ }^{75}$ one would extrapolate to $4.969 \AA$ at $0 \mathrm{~K}$, which is, indeed, very close to our optimized lattice constant.

Next, we turn to the electronic structure. In Fig. 4, we show the spin-resolved band structure and the spin- and orbital-resolved density of states calculated at the experimental lattice constant $(4.988 \AA$ ). At about -6 and $+6 \mathrm{eV}$ we find the localized majority and minority Gd $4 f$ state, respectively. In the vicinity of the Fermi energy, GdN exhibits a truly interesting electronic structure. Of particular interest are a direct and an indirect band gap, discussed extensively in the literature. The direct band gap accessible by optical measurements is located at the $\mathrm{X}$ point and amounts to $0.9 \mathrm{eV}$ for the majority spin channel and $1.5 \mathrm{eV}$ for the minority spin channel. The valence and the conduction states are separated by an indirect band gap $(\Gamma \rightarrow \mathrm{X})$; in the minority-spin states, this is a robust band gap of $1.5 \mathrm{eV}$, while in the majority-spin states this gap is tiny, only $0.01 \mathrm{eV}$. Thus we 


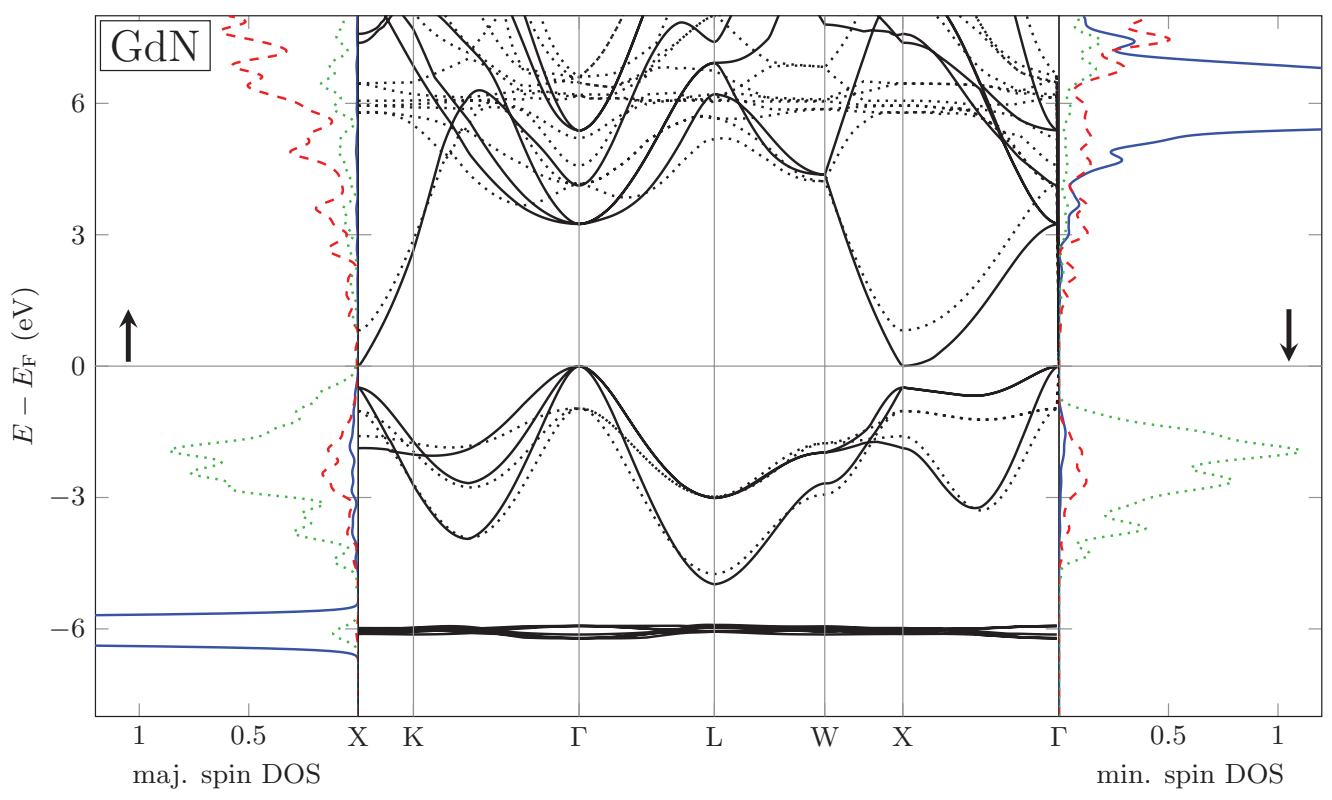

FIG. 4. (Color online) Band structure and density of states (DOS in states per eV) of GdN at the experimental lattice constant. The majority and minority bands are plotted as solid and dotted lines, respectively. The orbital-resolved DOS is shown on the left for majority and on the right for minority states. The solid blue line shows the Gd $4 f$ states, the red dashed line the Gd $5 d$ states, and the green dotted line the $\mathrm{N} 2 p$ states.

observe that GdN is in a narrow-gap semiconducting ground state with an almost vanishing indirect band gap $(\Gamma \rightarrow X)$ in the majority-spin direction, between the $\mathrm{N} 2 p$ states in the valence and the Gd $5 d$ states in the conduction band. This explains the different experimental reports disclosing $\mathrm{GdN}$ as a low-carrier semimetal or an insulator depending on small changes of the experimental circumstances.

Upon decreasing the lattice constant isotropically by just $0.021 \AA$ to the theoretically optimized value of $4.967 \AA$, we observe a transition to a half-metallic state or, more precisely, to a semimetallic state just for the majority states: a small portion of the $\mathrm{N} 2 p$ states at the $\Gamma$ point remains unoccupied, while the $\mathrm{Gd} 5 d$ band becomes partially occupied at the $\mathrm{X}$ point. If we define the "band gap" as the difference of these states, we formally get its value to be negative (see the band-transition energies in Table V). The described transition from a half-metallic to semiconducting state under isotropic strain was also observed by Duan et al., ${ }^{14}$ however at a much larger lattice constant of $5.63 \AA$. Our results suggest that, since the semiconductor to half-metal transition occurs so close to the equilibrium lattice parameter, the growth conditions, which influence the material properties such as the concentration of possible $\mathrm{N}$ vacancies or strain in the system, or the lattice expansion upon temperature changes play a decisive role in the transport properties of GdN.

Interesting physics can be expected also upon doping. If we $n$-dope into the conduction band or $p$-dope into the valence band, e.g., by use of $\mathrm{Eu}$, at a concentration where electrons or holes populate only majority states, we can obtain significant charge currents with $100 \%$ spin polarization. In the paramagnetic phase, minority and majority states converge to spin-degenerate valence and conduction states, and any spin polarization of the charge current disappears. At the same time, the band gap opens in the paramagnetic state, partly because of the thermal expansion and partly because of the averaged-out exchange potential felt by the electrons. This and a possible coupling of the conduction electrons or holes to the fluctuating $4 f$ moments $^{76}$ may change the conductivity by orders of magnitude when passing through the Curie temperature $\left(T_{\mathrm{C}}\right)$.

In Table $\mathrm{V}$, we list the band-transition energies calculated with our method at the experimental and at the optimized lattice constant for the ferromagnetic $\left(T<T_{\mathrm{C}}\right)$ and the paramagnetic $\left(T>T_{\mathrm{C}}\right)$ state. We find invariably larger band gaps for the paramagnetic state with no transition to a metallic state nearby. Our calculated band-transition energies compare well with the experimental data where available. The small indirect majority spin gap between $\Gamma$ and $\mathrm{X}$ of $0.01 \mathrm{eV}$ compares well with estimates of $0.05 \mathrm{eV}$ from Chantis et al. ${ }^{77}$ obtained using the quasiparticle self-consistent $G W$ method (QSGW) combined with their empirical rule to estimate band gaps in semiconductors. The transition energies obtained within the LSDA $+U$ method depend strongly on the choice of the parameter $U$. At the experimental lattice constant, we find similar transition energies as in the works of Larson et al. ${ }^{46}$ and Trodahl et al., ${ }^{68}$ where in the latter the parameter $U$ applied to the $4 f$ electrons was chosen to agree with the differences of the binding energies of the occupied and unoccupied $4 f$ levels as measured with the X-ray photoemission and inverse photoemission, respectively, in the Gd pnictides and the $U$ applied to $\mathrm{Gd} d$ states was chosen to reproduce the direct experimental band gap in the paramagnetic phase. In this way, the redshift of the direct band gap of $0.41 \mathrm{eV}$ going from the paramagnetic state to the ferromagnetic state is perfectly reproduced, while our parameter-free calculation gives $0.27 \mathrm{eV}$. The crossing of the conduction and the valence band at the $\mathrm{X}$ point was obtained with smaller values of $U,{ }^{14}$ whereas we find a direct band gap at $\mathrm{X}$ even below the Curie 
TABLE V. Comparison of our HSE results for GdN with those from LSDA+ $U$ and B3LYP calculations and experiment. The theoretical results are given for the optimized lattice constant, unless stated otherwise.

\begin{tabular}{|c|c|c|c|c|c|c|c|}
\hline & $\mathrm{HSE}^{\mathrm{a}}$ & HSE & $\mathrm{LSDA}+U^{\mathrm{b}}$ & $\mathrm{LSDA}+U^{\mathrm{c}}$ & $\mathrm{LSDA}+U^{\mathrm{a}, \mathrm{d}}$ & B3LYPe & Expt. \\
\hline Lattice constant $(\AA)$ & (4.988) & 4.967 & 4.92 & 5.08 & (4.988) & 5.05 & $4.988^{\mathrm{f}}$ \\
\hline Bulk modulus (GPa) & & 164 & & 150 & & 159 & $192^{\mathrm{g}}$ \\
\hline Magnetic moment $\left(\mu_{\mathrm{B}}\right)$ & 6.99 & 6.99 & & $6.93^{\mathrm{a}}$ & & 7.0 & $6.88^{\mathrm{h}}$ \\
\hline Direct gap at $\mathrm{X}(\mathrm{eV})\left(T<T_{\mathrm{C}}\right)$ & 0.90 & 0.85 & $-0.16^{\mathrm{i}}$ & & 0.91 & $1.18^{\mathrm{i}}$ & $0.90^{\mathrm{j}}$ \\
\hline Direct gap at $\mathrm{X}(\mathrm{eV})\left(T>T_{\mathrm{C}}\right)$ & 1.17 & 1.11 & $0.10^{\mathrm{i}}$ & $0.98^{\mathrm{a}}$ & 1.30 & $1.77^{\mathrm{i}}$ & $1.31^{\mathrm{j}}$ \\
\hline Indirect gap $\Gamma \rightarrow \mathrm{X}(\mathrm{eV})\left(T<T_{\mathrm{C}}\right)$ & 0.01 & -0.06 & $-0.45^{\mathrm{i}}$ & $0.14^{\mathrm{a}}$ & 0.43 & $0.72^{\mathrm{i}}$ & \\
\hline Indirect gap $\Gamma \rightarrow \mathrm{X}(\mathrm{eV})\left(T>T_{\mathrm{C}}\right)$ & 0.90 & 0.85 & $-0.13^{\mathrm{i}}$ & $0.69^{\mathrm{a}}$ & 0.98 & $1.47^{\mathrm{i}}$ & \\
\hline Position of majority $4 f$ peak $(\mathrm{eV})^{\mathrm{k}}$ & -6.00 & -6.00 & -7.8 & $-8.1^{\mathrm{a}, \mathrm{i}}$ & & $-6.3^{\mathrm{i}}$ & $-7.8^{1}$ \\
\hline Position of minority $4 f$ peak $(e V)^{\mathrm{k}}$ & 6.05 & 6.05 & 6.6 & $5.0^{\mathrm{a}, \mathrm{i}}$ & $4.8^{\mathrm{i}}$ & $5.5^{\mathrm{i}}$ & $5.5-6.1^{\mathrm{m}}$ \\
\hline
\end{tabular}

${ }^{\mathrm{a}}$ At the experimental lattice constant of $4.988 \AA$.

${ }^{\mathrm{b}}$ Reference 14; $U$ optimized for Gd bulk (Ref. 66).

${ }^{c}$ Reference 46; $U$ chosen to reproduce the experimental direct gap of paramagnetic GdN (Ref. 67).

${ }^{\mathrm{d}}$ Reference 68; $U$ chosen to reproduce the experimental direct gap of paramagnetic GdN.

${ }^{\mathrm{e}}$ Insulating solution of Ref. 23.

${ }^{\mathrm{f}}$ At room temperature; Ref. 69.

${ }^{\mathrm{g}}$ Reference 70.

${ }^{\text {h}}$ Reference 10

${ }^{\mathrm{i}}$ Extracted from the band structure.

${ }^{\mathrm{j}}$ Reference 68 .

${ }^{\mathrm{k}}$ Relative to the top of the valence band.

${ }^{1}$ Reference 11 .

${ }^{\mathrm{m}}$ Reference 71 measured for $\mathrm{Gd} X(X=\mathrm{P}, \mathrm{As}, \mathrm{Sb}$, and $\mathrm{Bi})$.

temperature $T_{\mathrm{C}}$. The calculation with $\mathrm{B} 3 \mathrm{LYP}^{23}$ yields three different solutions, where only the insulating one is similar to our result. The band gaps are significantly larger, which may be attributed to the larger optimized lattice constant.

The binding energy of the Gd $4 f$ majority band is also improved in the HSE scheme: the partial compensation of the self-interaction error leads to a pronounced shift of the localized $4 f$ states to larger binding energies. Calculation with the PBE functional yields a much too shallow $f$ majority band, located at $3.1 \mathrm{eV}$ below the Fermi energy; in HSE this band appears at a binding energy of $6.0 \mathrm{eV}$, much closer to its experimentally measured position at $7.8 \mathrm{eV} .{ }^{11}$ Furthermore, we note a very good agreement with the insulating B3LYP result, where the position of the $4 f$ peak is found at $6.3 \mathrm{eV} .{ }^{23}$ However, the agreement with experiment is not perfect. For the $f$ systems, a stronger mixing of nonlocal exchange would probably give a better result. As compared to PBE results, the unoccupied $4 f$ minority states shift toward higher energies $(6.05 \mathrm{eV})$, which is consistent with previous LSDA $+U$ and B3LYP calculations. ${ }^{14,23,46}$ The position of the unoccupied $4 f$ states agrees well with typical experimental results for the gadolinium pnictides between 5.5 and $6.1 \mathrm{eV}$ obtained with inverse photoemission spectroscopy. ${ }^{71}$

\section{Magnetic order and critical temperature}

The ground state of unconstrained bulk GdN is ferromagnetic (FM), with a Curie temperature of $58 \mathrm{~K}^{70}$ and a magnetic moment of $6.88 \mu_{\mathrm{B}}$ per $\mathrm{Gd}_{\text {atom }}{ }^{10}$ determined from the saturation magnetization at $1.2 \mathrm{~K}$. The total calculated magnetic moment is $7 \mu_{\mathrm{B}}$ per formula unit of which $6.99 \mu_{\mathrm{B}}$ comes from the Gd muffin-tin sphere. Our results are in good agreement with these observations (see the comparison of the theoretical and experimental values of the magnetic moment in Table V). We confirm the magnetic order and determine the critical temperature by mapping the total energies obtained from our HSE calculations for several magnetic structures onto the classical Heisenberg Hamiltonian

$$
\mathcal{H}=-\frac{1}{2} \sum_{i} \mathbf{S}_{i}\left(J_{1} \sum_{j=\mathrm{nn}} \mathbf{S}_{j}+J_{2} \sum_{j=\mathrm{nnn}} \mathbf{S}_{j}\right)
$$

including the nearest-neighbors (nn) and the next-nearestneighbors (nnn) interaction, where $J_{1}$ and $J_{2}$ are the respective coupling constants with normalized spin vectors $\mathbf{S}_{i}$ and $\mathbf{S}_{j}$. Positive and negative values of $J$ favor parallel and antiparallel spin alignment, respectively. The coupling constants are extracted from the differences of the total energies of the FM, and two types of antiferromagnetic (AFM) configurations characterized by planes of ferromagnetically ordered moments that are antiferromagnetically stacked along the crystallographic [001] or [111] directions (AFM-I and AFM-II, respectively). ${ }^{14}$ For the calculation of the AFM-I (AFM-II) phase we use a tetragonal $1 \times 1 \times 2$ (trigonal $\sqrt[3]{2} \times$ $\sqrt[3]{2} \times \sqrt[3]{2})$ unit cell containing two Gd atoms and calculate the FM state in the same unit cells, in order to guarantee reliable total energy differences. All the calculations are performed at the experimental lattice constant.

From the expressions

$$
\begin{gathered}
\Delta E_{I}=E_{\mathrm{AFM}, \mathrm{I}}-E_{\mathrm{FM}, \mathrm{I}}=8 J_{1}, \\
\Delta E_{I I}=E_{\mathrm{AFM}, \mathrm{II}}-E_{\mathrm{FM}, \mathrm{II}}=6 J_{1}+6 J_{2},
\end{gathered}
$$

the Heisenberg coupling constants $J_{1}$ and $J_{2}$ are easily obtained. We list them in Table VI, along with their values 
TABLE VI. Differences of total energies for different magnetic configurations [Eqs. (16) and (17)], the Heisenberg coupling constants, and the corresponding Curie temperatures within the meanfield approximation [Eq. (18)] and random phase approximation [Eq. (19)]. Energies and coupling constants are given in $\mathrm{meV}$ and the Curie temperatures in $\mathrm{K}$.

\begin{tabular}{lcccccc}
\hline \hline & $\Delta E_{I}$ & $\Delta E_{I I}$ & $J_{1}$ & $J_{2}$ & $T_{\mathrm{C}}^{\mathrm{MFA}}$ & $T_{\mathrm{C}}^{\mathrm{RPA}}$ \\
\hline This work & 8.8 & 7.6 & 1.09 & 0.17 & 55 & 42 \\
Duan et al. $^{\mathrm{a}}$ & 6.7 & 4.2 & 0.84 & -0.14 & 36 & 26 \\
Mitra et al. $^{\mathrm{b}}$ & 3.4 & 0.4 & 0.42 & -0.36 & 11 & 5 \\
\hline \hline
\end{tabular}

${ }^{\mathrm{a}}$ Reference 14 .

${ }^{\mathrm{b}}$ Reference 12

calculated in previous studies using an LSDA $+U$ method within an FLAPW ${ }^{14}$ and an LMTO basis set. ${ }^{12}$ Both coupling constants are positive, confirming the ferromagnetic nature of the ground state.

We use two approaches to estimate the Curie temperature. In the mean-field approximation (MFA),

$$
T_{\mathrm{C}}^{\mathrm{MFA}}=\frac{1}{3 k_{\mathrm{B}}}\left(12 J_{1}+6 J_{2}\right),
$$

we obtain $T_{\mathrm{C}}^{\mathrm{MFA}}=55 \mathrm{~K}$, very close to the experimental value of $58 \mathrm{~K}^{70}$ It is known, however, that the mean-field theory overestimates the Curie temperature. For comparison, we have also calculated the critical temperature by employing the random phase approximation (RPA) as described in Refs. 78 and 79, which is known to give results close to the Monte Carlo solution:

$$
T_{\mathrm{C}}^{\mathrm{RPA}}=\frac{1}{3 k_{\mathrm{B}}}\left[\int_{\mathrm{BZ}} \mathrm{d}^{3} q \frac{1}{J(\mathbf{0})-J(\mathbf{q})}\right]^{-1},
$$

where we evaluate the integral on a discrete mesh of $\mathbf{q}$ points within the Brillouin zone. $J(\mathbf{q})$ is the Fourier transform of the exchange coupling constants defined as

$$
J(\mathbf{q})=\sum_{\mathrm{nn}} J_{1} \mathrm{e}^{\mathrm{i} \mathbf{q} \cdot \mathbf{R}_{\mathrm{nn}}}+\sum_{\mathrm{nnn}} J_{2} \mathrm{e}^{\mathrm{i} \mathbf{q} \cdot \mathbf{R}_{\mathrm{nnn}}},
$$

where $\mathbf{R}_{\mathrm{nn}}$ and $\mathbf{R}_{\mathrm{nnn}}$ are the positions of the nearest and the next nearest neighbors, respectively. The resulting $T_{\mathrm{C}}^{\mathrm{RPA}}=42 \mathrm{~K}$ is roughly $30 \%$ smaller than the mean-field estimate. We consider these results as a sophisticated theoretical estimation of the Curie temperature that goes along with a few uncertainties, some of which are difficult to assess, such as the quality of HSE being an approximation to the true but unknown exchange and correlation functional and to a much lesser extent the adiabatic approximation inherent in applying the Heisenberg model. Easier to assess are the technically induced error estimates: (i) $\Delta E$ in Eqs. (16) and (17) are converged to about $1 \mathrm{meV}$, which translates to an uncertainty of $3 \mathrm{~K}$. (ii) Based on Monte Carlo calculations with two (as given in Table VI) and three nearest neighbors employing coupling constants published by Duan et al., ${ }^{14}$ which in both cases lead to the same Curie temperature of $28 \mathrm{~K}$, we estimate that the neglect of exchange interactions beyond next nearest neighbors leads to a maximum uncertainty of $1 \mathrm{~K}$. (iii) Employing our coupling constants (as given in Table VI), we find that the RPA result of $42 \mathrm{~K}$ approximates the numerically precise determination of the Curie temperature within the Heisenberg model obtained by Monte Carlo ( $45 \mathrm{~K}$ ) by $3 \mathrm{~K}$.

With these error estimates in mind, we compare our values to results of experimental studies, e.g. $T_{\mathrm{C}}=68,69,58$ or $37 \mathrm{~K}$ as reported by Granville et al., ${ }^{17}$ Khazen et al., ${ }^{80}$ Leuenberger et al., ${ }^{11}$ and Yoshitomi et al., ${ }^{81}$ which vary in value also depending on film thickness, strain, grain size, stoichiometry, and $\mathrm{N}$ vacancies. ${ }^{82,83} \mathrm{We}$ conclude that our results are in very good agreement with the experimental situation. Comparing our results to other theoretical values exhibited in Table VI we note that our coupling constants $J_{1}$ and $J_{2}$ obtained with HSE are significantly higher, which gives rise to a higher Curie temperature, in agreement with experiment. We observe that the increase of the coupling constants goes along with an increase of the Gd $4 f$ moment in the muffin-tin sphere by $90 \mathrm{~m} \mu_{\mathrm{B}}$ from $6.78 \mu_{\mathrm{B}}$ to $6.87 \mu_{\mathrm{B}}$, a decrease of the $\mathrm{Gd} 5 d$ moment by $20 \mathrm{~m} \mu_{\mathrm{B}}$ from 90 to $70 \mathrm{~m} \mu_{\mathrm{B}}$, and an increase of the $\mathrm{N} 2 p$ moment, which is aligned antiparallel to the $\mathrm{Gd} 4 f$ moment, by $20 \mathrm{~m} \mu_{\mathrm{B}}$, from -100 to $-120 \mathrm{~m} \mu_{\mathrm{B}}$, when HSE is compared to the PBE functional. The precise understanding of the relationship between the change of the moments and the coupling constants requires additional analysis that goes beyond the scope of the paper.

\section{CONCLUSION}

In this work, we have presented an implementation of the HSE hybrid functional, which contains a nonlocal screened exchange potential, within the FLAPW method as implemented in the FLEUR code. ${ }^{36}$ The calculation of the nonlocal exchange potential is realized by projecting the wave-function products onto the mixed product basis, reducing the six-dimensional integrations over the nonlocal interaction potential to vectormatrix-vector products, where the matrix must be calculated only once at the beginning of the self-consistent-field cycle.

We employ a sparse-matrix technique ${ }^{41}$ to evaluate the vector-matrix-vector products and incorporate the screening, i.e., the long-range part of the potential, in a separate step, where we exploit its fast converging Fourier series. This procedure allows constructing the nonlocal HSE potential from PBE0 up to machine precision at a negligible computational cost. We note that this approach is not restricted to the error function used in the HSE functional. In fact, our approach is quite general, it can be easily applied to arbitrarily screened interaction potentials.

The results for lattice constants and band-transition energies obtained within our method show excellent agreement with previous results obtained with the $\mathrm{PAW}^{35}$ and Gaussian-based ${ }^{52}$ methods. We have confirmed the finding of Paier et al. ${ }^{35}$ that the $\mathbf{k}$-point convergence within HSE is comparable to the conventional local PBE functional, whereas in PBE0 much larger k-point meshes are necessary.

In addition, we have calculated the properties of the rareearth compound GdN. There is an ongoing discussion whether the ground state is insulating or metallic. In fact, within the HSE functional the ground state is very close to a phase transition; we observe a tiny indirect band gap at the experimental lattice constant at room temperature, which vanishes at the theoretically optimized $0 \mathrm{~K}$ lattice constant - the compound becomes half-metallic. The experimentally known band 
transitions are in good agreement with our theoretical results. Furthermore, we have calculated the coupling constants for the Heisenberg spin Hamiltonian from total-energy differences of ferromagnetic and antiferromagnetic configurations. The resulting Curie temperature of $42 \mathrm{~K}$ evaluated in the randomphase approximation is in good agreement with the experimental value of $58 \mathrm{~K}$ and gives confidence in the energetics obtained by HSE for different magnetic phases. From this we conclude that the HSE functional has the potential to describe the properties of rare-earth chalcogenides without the need for employing a Hubbard $U$ parameter. We encourage the community to make use of the potential of the HSE functional to ex- plore the more subtle properties of the rare-earth chalcogenides such as the physics due to strain, dopands, or heterostructures.

\section{ACKNOWLEDGMENTS}

We would like to thank Walter Lambrecht for fruitful discussions on GdN. We gratefully acknowledge the funding by the Young Investigators Group Programme of the Helmholtz Association ("Computational Nanoferronics Laboratory," Contract VH-NG-409) and by the Deutsche Forschungsgemeinschaft through the Priority Program 1145. *m.schlipf@fz-juelich.de

${ }^{1}$ P. Hohenberg and W. Kohn, Phys. Rev. 136, B864 (1964).

${ }^{2}$ W. Kohn and L. J. Sham, Phys. Rev. 140, A1133 (1965).

${ }^{3}$ U. von Barth and L. Hedin, J. Phys. C 5, 1629 (1972).

${ }^{4}$ O. Gunnarsson and B. I. Lundqvist, Phys. Rev. B 13, 4274 (1976).

${ }^{5}$ D. M. Ceperley and B. J. Alder, Phys. Rev. Lett. 45, 566 (1980).

${ }^{6}$ J. P. Perdew and Y. Wang, Phys. Rev. B 33, 8800 (1986).

${ }^{7}$ J. P. Perdew, K. Burke, and M. Ernzerhof, Phys. Rev. Lett. 77, 3865 (1996).

${ }^{8}$ E. Fermi and E. Amaldi, Le orbite $\infty$ s degli elementi, Memorie della Reale accademia d'Italia: Classe di scienze siche, matematiche e naturali (Reale Accademia d'Italia, Rome, 1934).

${ }^{9}$ J. P. Perdew and A. Zunger, Phys. Rev. B 23, 5048 (1981).

${ }^{10}$ D. X. Li, Y. Haga, H. Shida, and T. Suzuki, Physica B 199-200, 631 (1994).

${ }^{11}$ F. Leuenberger, A. Parge, W. Felsch, K. Fauth, and M. Hessler, Phys. Rev. B 72, 014427 (2005).

${ }^{12}$ C. Mitra and W. R. L. Lambrecht, Phys. Rev. B 78, 134421 (2008).

${ }^{13}$ A. Sharma and W. Nolting, Phys. Rev. B 81, 125303 (2010).

${ }^{14}$ C.-G. Duan, R. F. Sabiryanov, J. Liu, W. N. Mei, P. A. Dowben, and J. R. Hardy, Phys. Rev. Lett. 94, 237201 (2005); C.-G. Duan, R. F. Sabiryanov, W. N. Mei, P. A. Dowben, S. S. Jaswal, and E. Y. Tsymbal, Appl. Phys. Lett. 88, 182505 (2006); J. Phys. Condens. Matter. 19, 315220 (2007).

${ }^{15}$ P. Wachter and E. Kaldis, Solid State Commun. 34, 241 (1980).

${ }^{16}$ J. Q. Xiao and C. L. Chien, Phys. Rev. Lett. 76, 1727 (1996).

${ }^{17}$ S. Granville, B. J. Ruck, F. Budde, A. Koo, D. J. Pringle, F. Kuchler, A. R. H. Preston, D. H. Housden, N. Lund, A. Bittar, G. V. M. Williams, and H. J. Trodahl, Phys. Rev. B 73, 235335 (2006).

${ }^{18}$ B. M. Ludbrook, I. L. Farrell, M. Kuebel, B. J. Ruck, A. R. H. Preston, H. J. Trodahl, L. Ranno, R. J. Reeves, and S. M. Durbin, J. Appl. Phys. 106, 063910 (2009).

${ }^{19}$ M. Scarpulla, C. Gallinat, S. Mack, J. Speck, and A. Gossard, J. Cryst. Growth 311, 1239 (2009).

${ }^{20}$ W. R. L. Lambrecht, Phys. Rev. B 62, 13538 (2000).

${ }^{21}$ D. B. Ghosh, M. De, and S. K. De, Phys. Rev. B 72, 045140 (2005).

${ }^{22}$ C. M. Aerts, P. Strange, M. Horne, W. M. Temmerman, Z. Szotek, and A. Svane, Phys. Rev. B 69, 045115 (2004).

${ }^{23}$ K. Doll, J. Phys. Condens. Matter 20, 075214 (2008).

${ }^{24}$ V. I. Anisimov, F. Aryasetiawan, and A. I. Lichtenstein, J. Phys. Condens. Matter 9, 767 (1997).

${ }^{25}$ P. H. Dederichs, S. Blügel, R. Zeller, and H. Akai, Phys. Rev. Lett. 53, 2512 (1984).
${ }^{26}$ M. Cococcioni and S. de Gironcoli, Phys. Rev. B 71, 035105 (2005).

${ }^{27}$ E. Şaşığlu, C. Friedrich, and S. Blügel, Phys. Rev. B 83, 121101 (2011).

${ }^{28}$ A. Rohrbach, J. Hafner, and G. Kresse, J. Phys. Condens. Matter 15, 979 (2003).

${ }^{29}$ J. Muscat, A. Wander, and N. Harrison, Chem. Phys. Lett. 342, 397 (2001).

${ }^{30}$ E. N. Brothers, A. F. Izmaylov, J. O. Normand, V. Barone, and G. E. Scuseria, J. Chem. Phys. 129, 011102 (2008).

${ }^{31}$ A. D. Becke, J. Chem. Phys. 98, 1372 (1993); 98, 5648 (1993).

${ }^{32}$ J. P. Perdew, M. Ernzerhof, and K. Burke, J. Chem. Phys. 105, 9982 (1996).

${ }^{33}$ J. Heyd, G. E. Scuseria, and M. Ernzerhof, J. Chem. Phys. 118, 8207 (2003).

${ }^{34}$ J. Paier, R. Hirschl, M. Marsman, and G. Kresse, J. Chem. Phys. 122, 1 (2005).

${ }^{35}$ J. Paier, M. Marsman, K. Hummer, G. Kresse, I. C. Gerber, and J. G. Ángyán, J. Chem. Phys. 124, 154709 (2006); 124, 249901 (2006).

${ }^{36}$ http://www.flapw.de

${ }^{37}$ E. Wimmer, H. Krakauer, M. Weinert, and A. J. Freeman, Phys. Rev. B 24, 864 (1981).

${ }^{38}$ M. Weinert, E. Wimmer, and A. J. Freeman, Phys. Rev. B 26, 4571 (1982).

${ }^{39}$ H. J. F. Jansen and A. J. Freeman, Phys. Rev. B 30, 561 (1984).

${ }^{40}$ F. Tran, P. Blaha, K. Schwarz, and P. Novák, Phys. Rev. B 74, 155108 (2006).

${ }^{41}$ M. Betzinger, C. Friedrich, and S. Blügel, Phys. Rev. B 81, 195117 (2010).

${ }^{42}$ F. Tran and P. Blaha, Phys. Rev. B 83, 235118 (2011).

${ }^{43}$ M. Weinert, J. Math. Phys. 22, 2433 (1981).

${ }^{44}$ C. Friedrich, A. Schindlmayr, and S. Blügel, Comput. Phys. Commun. 180, 347 (2009).

${ }^{45}$ P. Larson and W. R. L. Lambrecht, Phys. Rev. B 74, 085108 (2006).

${ }^{46}$ P. Larson, W. R. L. Lambrecht, A. Chantis, and M. van Schilfgaarde, Phys. Rev. B 75, 045114 (2007).

${ }^{47}$ J. Harris and R. O. Jones, J. Phys. F 4, 1170 (1974).

${ }^{48}$ D. C. Langreth and J. P. Perdew, Phys. Rev. B 15, 2884 (1977).

${ }^{49}$ A. D. Becke, J. Chem. Phys. 104, 1040 (1996).

${ }^{50}$ B. G. Janesko, T. M. Henderson, and G. E. Scuseria, Phys. Chem. Chem. Phys. 11, 443 (2009).

${ }^{51}$ J. Heyd and G. E. Scuseria, J. Chem. Phys. 120, 7274 (2004). 
${ }^{52}$ A. V. Krukau, O. A. Vydrov, A. F. Izmaylov, and G. E. Scuseria, J. Chem. Phys. 125, 224106 (2006).

${ }^{53}$ A. Seidl, A. Görling, P. Vogl, J. A. Majewski, and M. Levy, Phys. Rev. B 53, 3764 (1996).

${ }^{54}$ C. Friedrich, S. Blügel, and A. Schindlmayr, Phys. Rev. B 81, 125102 (2010).

${ }^{55}$ J. Heyd and G. E. Scuseria, J. Chem. Phys. 121, 1187 (2004).

${ }^{56}$ Numerical Data and Functional Relationships in Science and Technology, Group III Condensed Matter, edited by K.-H. Hellwege, O. Madelung, M. Schulz, and H. Weiss (Springer-Verlag, New York, 1982), Vols. 17 and 22.

${ }^{57}$ T. Chiang and F. J. Himpsel, in Electronic Structure of Solids: Photoemission Spectra and Related Data, Group III: Condensed Matter, edited by A. Goldmann and E.-E. Koch (Springer-Verlag, Berlin, 1989), Vol. 23a.

${ }^{58}$ J. E. Ortega and F. J. Himpsel, Phys. Rev. B 47, 2130 (1993).

${ }^{59}$ M. Welkowsky and R. Braunstein, Phys. Rev. B 5, 497 (1972).

${ }^{60}$ R. Hulthén and N. G. Nilsson, Solid State Commun. 18, 1341 (1976).

${ }^{61} \mathrm{~S}$. Adachi, Optical Properties of Crystalline and Amorphous Semiconductors: Numerical Data and Graphical Information (Kluwer Academic, Dordrecht, 1999).

${ }^{62}$ R. T. Poole, J. Liesegang, R. C. G. Leckey, and J. G. Jenkin, Phys. Rev. B 11, 5190 (1975).

${ }^{63}$ R. J. Magyar, A. Fleszar, and E. K. U. Gross, Phys. Rev. B 69, 045111 (2004).

${ }^{64}$ F. Murnaghan, Proc. Nat. Acad. Sci. USA 30, 244 (1944).

${ }^{65}$ M. Betzinger, C. Friedrich, S. Blügel, and A. Görling, Phys. Rev. B 83, 045105 (2011).

${ }^{66}$ B. N. Harmon, V. P. Antropov, A. I. Liechtenstein, I. V. Solovyev, and V. I. Anisimov, J. Phys. Chem. Solids 56, 1521 (1995); proceedings of the 1994 Conference on Magneto-optic Materials.

${ }^{67} \mathrm{~F}$. Hullinger, in Handbook on the Physics and Chemistry of Rare Earths, edited by K. A. Gschneidner Jr. and L. R. Eyring (NorthHolland Physics, New York, 1979), Vol. 4, pp. 153-236.
${ }^{68}$ H. J. Trodahl, A. R. H. Preston, J. Zhong, B. J. Ruck, N. M. Strickland, C. Mitra, and W. R. L. Lambrecht, Phys. Rev. B 76, 085211 (2007).

${ }^{69}$ R. C. Brown and N. J. Clark, J. Inorg. Nucl. Chem. 36, 2507 (1974).

${ }^{70}$ U. Rössler and D. Strauch, Group IV Elements, IV-IV and III-V Compounds, Group III Condensed Matter (Springer-Verlag, Berlin, 2001), Vol. 41a1a.

${ }^{71}$ H. Yamada, T. Fukawa, T. Muro, Y. Tanaka, S. Imada, S. Suga, D.-X. Li, and T. Suzuki, J. Phys. Soc. Jpn. 65, 1000 (1996).

${ }^{72}$ D. Singh, Phys. Rev. B 43, 6388 (1991).

${ }^{73}$ E. E. Krasovskii, Phys. Rev. B 56, 12866 (1997).

${ }^{74}$ C. Friedrich, A. Schindlmayr, S. Blügel, and T. Kotani, Phys. Rev. B 74, 045104 (2006).

${ }^{75}$ F. Lévy, Phys. Kondens. Mater. 10, 71 (1969).

${ }^{76}$ M. Arnold and J. Kroha, Phys. Rev. Lett. 100, 046404 (2008).

${ }^{77}$ A. N. Chantis, M. van Schilfgaarde, and T. Kotani, Phys. Rev. B 76, 165126 (2007).

${ }^{78}$ N. N. Bogoliubov and S. V. Tyablikov, Dokl. Akad. Nauk SSSR 126, 53 (1959) [Sov. Phys.-Dokl. 4, 589 (1959)].

${ }^{79}$ U. Nowak, Matter and Materials, edited by S. Blügel, T. Brückel, and C. M. Schneider (Forschungszentrum Jülich, Jülich, 2005), Vol. 26, p. A3.1.

${ }^{80} \mathrm{~K}$. Khazen, H. J. von Bardeleben, J. L. Cantin, A. Bittar, S. Granville, H. J. Trodahl, and B. J. Ruck, Phys. Rev. B 74, 245330 (2006).

${ }^{81}$ H. Yoshitomi, S. Kitayama, T. Kita, O. Wada, M. Fujisawa, H. Ohta, and T. Sakurai, Phys. Rev. B 83, 155202 (2011).

${ }^{82}$ K. Senapati, T. Fix, M. E. Vickers, M. G. Blamire, and Z. H. Barber, Phys. Rev. B 83, 014403 (2011).

${ }^{83}$ A. Punya, T. Cheiwchanchamnangij, A. Thiess, and W. R. L. Lambrecht, MRS online proceedings 1290, mrsf10-1290-i04-04 (2011). 